\title{
The $\mathrm{Ca}^{2+}$-Permeable Cation Transient Receptor Potential TRPV3 Channel: An Emerging Pivotal Target for Itch and Skin Diseases
}

\author{
Gongxin Wang and KeWei Wang \\ Department of Pharmacology, Qingdao University School of Pharmacy and Institute of Innovative Drugs, Qingdao University, \\ Qingdao, Shandong Province, China
}

Received December 20, 2016; accepted March 31, 2017

\section{ABSTRACT}

Temperature-sensitive transient receptor potential (TRP) channels such as TRPA1 and TRPV1 have been identified as downstream ion channel targets in the transduction of itch. As a member of the temperature-sensitive TRP family, the $\mathrm{Ca}^{2+}$-permeable nonselective cation channel TRPV3 is expressed abundantly in skin keratinocytes. Recent identification of gain-of-function mutations of human TRPV3 from patients with Olmsted syndrome, which is characterized by severe itching and palmoplantar and periorificial keratoderma, unveils its crucial role in chronic itch and skin diseases. In this review, we will focus on recent progress made in the understanding of TRPV3 that emerges as an attractive target for developing effective antipruritic therapy for chronic itch or skin-related diseases.

\section{Introduction}

Itch (also known as pruritus) is an unpleasant sensation of the skin, provoking the desire or reflex to scratch. Although acute itch is often caused in the local affected skin by pruritogens (e.g., histamine, allergens, inflammatory mediators, and drugs), chronic itch that impairs quality of life can be an indicator of widespread symptoms associated with cancers and inflammatory skin, infectious, immune, and liver diseases. With the exception of histamine-induced itch, there is generally no accepted treatment of chronic itch, which is still an unmet medical need. The lack of effective itch therapies is primarily attributed to the insufficient understanding of the crucial target(s) that underlies the pathogenesis of itch. Previous findings have shown that several subtypes of transient receptor potential (TRP) channels play important roles in different types of itch induced by pruritogens in rodents (see Zhang, 2015). However, the recent identification of gain-of-function mutations in human TRPV3 from patients with Olmsted syndrome characterized by severe itching and skin diseases unveils the crucial role of TRPV3 channels in itch signaling (Fig. 1). As a result, TRPV3 emerges as an

This research was supported by the National Natural Sciences Foundation of China [Grant 81573410 (to K.W.)], the Ministry of Science and Technology of China [Grant 2014ZX09507003-006-004 (to K.W.)], and the Natural Sciences Foundation of Shandong Province [Grant ZR2015QL008 (to K.W.)].

https://doi.org/10.1124/mol.116.107946. attractive target for developing antipruritic therapy in chronic itch or skin-related diseases.

The superfamily of TRP channels is composed of 28 mammalian or 27 human members that can be subdivided in six subfamilies, including the TRP subfamily A (ankyrin, TRPA), the TRP subfamily C (canonical, TRPC), the TRP subfamily M (melastatin, TRPM), the TRP polycystin subfamily (TRPP), the TRP mucolipin subfamily (TRPML), and the TRP subfamily V (vanilloid, TRPV) (Holzer and Izzo, 2014). Most TRP channels as homo- or probably heterotetramers permeate cations through central nonselective cation entry pores that are symmetrically located in the plasma membrane (Moiseenkova-Bell and Wensel, 2009). In general, TRP channels open and close in response to changes in temperature, chemical stimulation, ligand binding, and weak membrane depolarization (Nilius et al., 2005; Klein et al., 2015; Laing and Dhaka, 2016).

\section{The Properties of TRPV3 Channels}

Among the temperature-sensitive or thermoTRPV1-4 channels, TRPV3 exhibits distinct temperature activation at $>33^{\circ} \mathrm{C}$ compared with TRPV1, TRPV2, and TRPV4, which are activated at $>43^{\circ} \mathrm{C},>52^{\circ} \mathrm{C}$, and $>30^{\circ} \mathrm{C}$, respectively (FerrerMontiel et al., 2012). As a thermosensor, TRPV3 appears as a surprise, in that it is poorly detected in the dorsal root

ABBREVIATIONS: 2-APB, 2-aminoethoxydiphenyl borate; 17R-RvD1, 17(R)-resolvin D1; AD, atopic dermatitis; AEW, acetone/ether/water; ARD, ankyrin repeat domain; CaM, calmodulin; DRG, dorsal root ganglion; EGFR, epidermal growth factor receptor; HEK293, human embryonic kidney 293; PGE2, prostaglandin E2; PIP 2 , phosphatidylinositol 4,5-biphosphate; TG, transglutaminase; TGF- $\alpha$, transforming growth factor- $\alpha$; TRP, transient receptor potential; W-7, N-(6-aminohexyl)-5-chloro-1-naphthalenesulfonamide hydrochloride. 


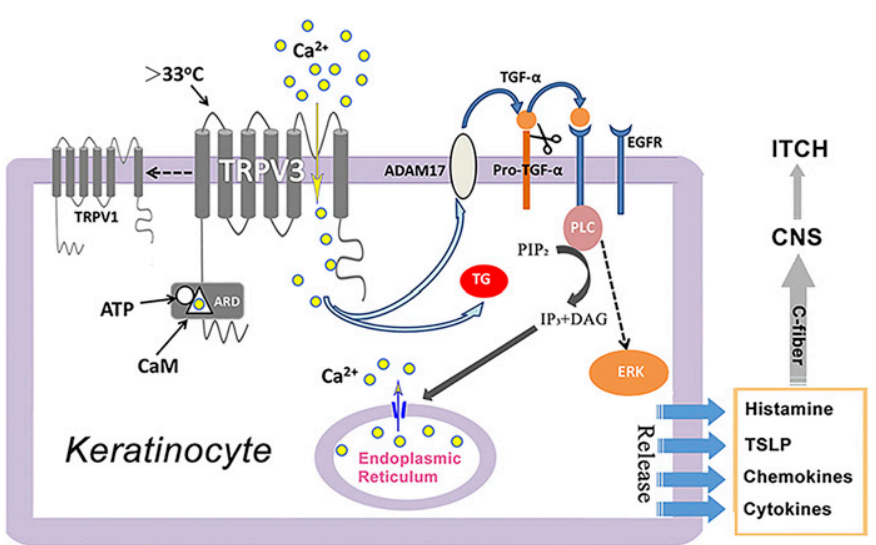

Fig. 1. Proposed role of TRPV3 in itch signaling and hair growth. TRPV3 is abundantly expressed in keratinocytes, the predominant cell type comprising the layers of the epidermis in the skin. Keratinocytes release numerous inflammatory molecules such as histamine, TSLP, chemokines, and cytokines, accounting for enhanced pruriceptor sensitivity under chronic itch conditions. The TRPV3 channel is activated by mechanisms that include elevated intracellular acidification (protons), temperature, activators, and other unidentified cellular events, resulting in an elevation of $\mathrm{Ca}^{2+}$-dependent production and release of TGF- $\alpha$ or other EGFR ligands. TGF- $\alpha$ in turn stimulates EGFR, which physically associates with TRPV3 to form a signaling complex, and consequently sensitizes the responses of TRPV3 to endogenous activation. Thus, a positive feedback loop is formed between TRPV 3 and TGF- $\alpha /$ EGFR, likely resulting in terminal differentiation of suprabasal keratinocytes that are actively participated in reepithelialization, wound closure, and hair morphogenesis. Coexpression of TRPV3 with TRPV1 enhances the capsaicin- or proton-evoked rise of intracellular $\mathrm{Ca}^{2+}$ concentrations, suggesting a physical association between the two proteins. Binding of ATP to conserved sites in the N-terminal ARD domains shared by TRPV3 and TRPV1 channels also suggests functional interactions between the two channels. ADAM17, metalloprotease ADAM17; ERK, extracellular signal-regulated kinase; CNS, central nervous system; DAG, diacylglycerol; $\mathrm{IP}_{3}$, inositol trisphosphate; PLC, phospholipase C; TSLP, thymic stromal lymphopoietin.

ganglion (DRG) or the trigeminal ganglion (TG) sensory neurons in rodents. Reports suggest that keratinocytes may release diffusible molecules that in turn can activate free nerve endings of neighboring DRG neurons when TRPV3 is activated by temperatures of $>32^{\circ} \mathrm{C}$ in the skin (Mandadi et al., 2009; Gifford et al., 2012). This hypothesis was tested by coculturing TRPV1-deficient DRG neurons with keratinocytes (Mandadi et al., 2009). It was shown that the cytosolic concentration of $\mathrm{Ca}^{2+}$ in DRG sensory neurons was increased upon heating only when they were cocultured with keratinocytes, and the increase of intracellular calcium can be inhibited by suramin, $\mathrm{P} 2$ purinergic antagonists, and pyridoxalphosphate6 -azophenyl-2',4'-disulfonic acid tetrasodium salt (Mandadi et al., 2009). In cocultured keratinocytes and human embryonic kidney 293 (HEK293) cells, keratinocytes release ATP upon heating; this response is reduced in TRPV3-knockout mouse keratinocytes (Mandadi et al., 2009), suggesting that ATP can function as a messenger molecule for thermotransduction mediated by TRPV3 in the skin. Keratinocytes release ATP, and ATP accumulation in the interstitial fluid has been detected using microdialysis (Mandadi et al., 2009; Gifford et al., 2012).

TRPV3 belongs to the TRPV channel subfamily comprising six members. In humans, the gene for TRPV3, which contains 18 exons, is located on chromosome $17 \mathrm{p} 13$ in close proximity to the TRPV1 gene. Similar to human TRPV3, the mouse TRPV3 gene also has 18 exons and is located in chromosome 11B4 (Peier et al., 2002; Smith et al., 2002; Xu et al., 2002). The complete open reading frame of the mouse or human TRPV3 gene is $2373 \mathrm{bp}$, encoding for a polypeptide containing 791 amino acids. However, the TRPV3 gene may be differentially spliced, yielding 790, 791, and 765 amino acid variants in humans (Smith et al., 2002; Xu et al., 2002). It has been shown by in situ hybridization and reverse transcription polymerase chain reaction analysis that TRPV3 is most abundantly expressed in keratinocytes of the skin as well as in oral and nasal epithelia in both humans and mice (Moqrich et al., 2005; Xu et al., 2006; Ahmed et al., 2009). TRPV3 is also detected in the brain, spinal cord, DRG, TG, and testes in humans (Peier et al., 2002; Smith et al., 2002; Xu et al., 2002) but is not highly expressed in the DRG or TG in rodents (Peier et al., 2002; Moqrich et al., 2005).

There is an ankyrin repeat domain (ARD) in the intracellular $\mathrm{N}$ terminus in all six members of the TRPV subfamily. Each ankyrin repeat is typically composed of 33 residues that function as a motif that is primarily involved in subunitsubunit interactions. Although the overall structure of TRPV3-ARD is similar to that of ARDs from other members of the TRPV subfamily, TRPV3 exhibits a unique bent finger 3 loop that is stabilized by hydrogen bonds and hydrophobic packing. This loop is not as flexible as those in other known TRPV-ARD structures, including TRPV1, TRPV2, TRPV4, and TRPV6 (Jin et al., 2006; McCleverty et al., 2006; Lishko et al., 2007; Phelps et al., 2008; Auer-Grumbach et al., 2010; Inada et al., 2012).

\section{Chemical Activation and Inhibition of TRPV3}

Since TRPV3 was discovered, a number of natural and synthetic compounds targeting TRPV3 have been identified (Table 1). However, all of the known compounds are nonspecific and their effective concentrations are relatively high. Among these compounds, 2-aminoethoxydiphenyl borate (2APB) is the most commonly used activator of TRPV3 and, to a lesser extent, TRPV1 and TRPV2, but this compound does not activate TRPV4, TRPV5, and TRPV6 (Hu et al., 2004). 2-APB also inhibits the activation of TRPM8 and TRPC 6 evoked by menthol and 1-oleolyl-2-acetyl-sn-glycerol, respectively (Hu et al., 2004). It was initially reported that 2-APB functions as an inositol trisphosphate receptor inhibitor; it was subsequently shown to also block store-operated $\mathrm{Ca}^{2+}$ channels (Ma et al., 2000). The action of 2-APB on TRP channels is complicated, in that it also causes an inhibition of TRPV3 at higher concentrations ( $>300 \mu \mathrm{M})(\mathrm{Hu}$ et al., 2004). Similarly, it was reported that some 2-APB analogs are either activators or inhibitors of TRPV3 (Deering-Rice et al., 2014). The sensitivity of mouse TRPV3 to 2-APB, but not to camphor or voltage, is critically determined by a cytoplasmic N-terminal residue (H426) and a $\mathrm{C}$-terminal residue (R696) in the channel (Hu et al., 2009).

Some natural plant products, such as camphor, eugenol, carvacrol, thymol, menthol, cinnamaldehyde, and citral, can activate TRPV3 (Xu et al., 2006; Stotz et al., 2008; Sherkheli et al., 2009, 2013; Earley et al., 2010; Ortar et al., 2012; Klein et al., 2013). The majority of these natural compounds are commonly known as flavoring ingredients, allergens, and/or skin sensitizers. Besides activating TRPV3 directly, 2-APB even at subthreshold concentrations also promotes TRPV3 current sensitization to heat (Chung et al., 2004). Likewise, TRPV3 can be potentiated or sensitized by unsaturated fatty 
TABLE 1

Known compounds targeting TRPV3 channels

\begin{tabular}{|c|c|c|c|c|}
\hline Compound & Activate & Inhibit & Description/Use & Reference \\
\hline 2-APB & TRPV1/TRPV2/TRPV3/TRPV6 & TRPC6/TRPM8 & $\begin{array}{l}\text { Inhibitor of store-operated } \mathrm{Ca}^{2+} \text { influx } \\
\text { and } \mathrm{IP}_{3} \text {-mediated } \mathrm{Ca}^{2+} \text { release; } \\
\text { common regulator of TRP channels }\end{array}$ & $\begin{array}{l}\text { Chung et al., 2004; Hu } \\
\text { et al., } 2004\end{array}$ \\
\hline Camphor & TRPV1/TRPV3 & TRPA1 & $\begin{array}{l}\text { Modulate sensations of warmth in } \\
\text { humans }\end{array}$ & $\begin{array}{l}\text { Moqrich et al., 2005; Xu } \\
\text { et al., } 2005\end{array}$ \\
\hline Menthol & TRPV3/TRPM8/TRPA1 & TRPA1 & $\begin{array}{l}\text { Cooling effect; modulation of warm } \\
\text { sensation }\end{array}$ & Macpherson et al., 2006 \\
\hline Cinnamaldehyde & TRPV3/TRPA1 & TRPM8 & Used as a spice & Macpherson et al., 2006 \\
\hline Carvacrol & TRPV3/TRPA1 & & $\begin{array}{l}\text { Rapidly desensitizes TRPA1; present } \\
\text { in oregano, which is used as a spice }\end{array}$ & Xu et al., 2006 \\
\hline Eugenol & TRPV3/TRPA1 & & $\begin{array}{l}\text { Present in clove, which is used as a } \\
\text { spice; lingual irritation; innocuous } \\
\text { warmth and pain in response to } \\
\text { heat }\end{array}$ & $\begin{array}{l}\text { Schöll and Jensen-Jarolim, } \\
\text { 2004; Xu et al., } 2006\end{array}$ \\
\hline Thymol & TRPV3/TRPA1/TRPM8 & & $\begin{array}{l}\text { Derived from thyme, which is used as } \\
\text { a spice }\end{array}$ & $\mathrm{Xu}$ et al., 2006 \\
\hline Incensole acetate & TRPV3 & & $\begin{array}{l}\text { A novel anxiolytic and antidepressive } \\
\text { agent }\end{array}$ & Moussaieff et al., 2008 \\
\hline FPP & TRPV3 & & $\begin{array}{l}\text { First identified endogenous TRPV3 } \\
\text { activator; causes nociception }\end{array}$ & Bang et al., 2010 \\
\hline IPP & & TRPV3/TRPA1 & $\begin{array}{l}\text { Endogenous inhibitor; a precursor } \\
\text { molecule for FPP synthesis in the } \\
\text { mevalonate pathway }\end{array}$ & Bang et al., 2011 \\
\hline Drofenine & TRPV3 & & $\begin{array}{l}\text { Antispasmodic agent; 2-APB analog; } \\
\text { improved selectivity for TRPV3 }\end{array}$ & Deering-Rice et al., 2014 \\
\hline DPBA & TRPV1/TRPV2/TRPV3/TRPV4 & & 2-APB structural analogs & Chung et al., 2005 \\
\hline DPTHF & & $\begin{array}{l}\text { TRPV1/TRPV2/ } \\
\text { TRPV3 }\end{array}$ & 2-APB structural analogs & Chung et al., 2005 \\
\hline Nicotinic acid & TRPV1/TRPV3 & TRPV2/TRPV4 & $\begin{array}{l}\text { Used to treat dyslipidemias but causes } \\
\text { a side effect of cutaneous } \\
\text { vasodilation, commonly called } \\
\text { flushing }\end{array}$ & Ma et al., 2015 \\
\hline 17R-RvD1 & & TRPV3 & Proresolving lipid & Bang et al., 2012 \\
\hline Icilin & TRPM8 & TRPV3 & Super cooling agent & Sherkheli et al., 2012 \\
\hline
\end{tabular}

DPBA, diphenylboronic anhydride; DPTHF, diphenyltetrahydrofuran; FPP, farnesyl pyrophosphate; $\mathrm{IP}_{3}$, inositol trisphosphate; IPP, isopentenyl pyrophosphate.

acids as well as by cholesterol (Hu et al., 2006; Klein et al., 2014). We also found that glycolic acid can strongly activate the TRPV3 channel (Cao et al., 2012). This activation is mediated by intracellular protons that act on residue $\mathrm{H} 426$, located at the distal $\mathrm{N}$ terminus, which is the same site shown to be important for 2-APB action on TRPV3 (Cao et al., 2012). Other cytoplasmic residues in TRPV3, such as L508, D512, $\mathrm{S} 518$, or A520, have also been reported to play a role in proton sensing (Fig. 2) (Gao et al., 2016). This novel gating mechanism, in which TRPV3 is directly activated by intracellular acidification, likely explains the cosmetic effect of $\alpha$-hydroxyl acids on keratinization of the skin (Cao et al., 2012).

To date, there is a lack of specific TRPV3 antagonists, although there are several TRPV3 antagonists including the anti-inflammatory lipid mediator $17(R)$-resolvin D1 (17RRvD1) (Bang et al., 2012), the 2-APB structural analog diphenyltetrahydrofuran (Chung et al., 2005), the TRPM8 agonist icilin (Sherkheli et al., 2012), and isopentenyl pyrophosphate (Bang et al., 2011). However, these TRPV3 antagonists are not specific. Nevertheless, a number of TRPV3 modulators are currently under development as possible therapeutic agents with potential use in conditions including psoriasis, itch, dermatitis, hirsutism, and pain. Therefore, it is necessary to identify selective TRPV3 modulators (agonists or antagonists) that can be used either as tools to study the channel physiology or as lead compounds for validation of TRPV3 as a therapeutic target in the treatment of itch or skinrelated diseases.

\section{Regulation and Gating of TRPV3}

TRPV3 is shown to be sensitized by repetitive ligand stimulations (Xu et al., 2002; Xiao et al., 2008a). Both extracellular and intracellular calcium ions play an important role in TRPV3 sensitization to repetitive stimulations by 2-APB or other stimuli, causing a slow activation at positive potential and a strong deactivation at negative potentials (Xiao et al., 2008a). The sensitization of TRPV3 to repetitive stimuli is likely dependent on a reduction of extracellular $\mathrm{Ca}^{2+}$ that binds to residues such as Asp641 at the pore loop, since conserved acidic residues at the equivalent positions of rat TRPV1 (Asp646) and mouse TRPV4 (Asp682) seem to play a major role in binding to divalent cations and are vital for the inhibitory activity of ruthenium red (García-Martínez et al., 2000). More interestingly, the sensitization of TRPV3 to repetitive ligand stimulations can be abolished by calmodulin (CaM) inhibitors such as calmidazolium, ophiobolin A, and W7 (N-(6-aminohexyl)-5-chloro-1-naphthalenesulfonamide hydrochloride), suggesting that $\mathrm{Ca}^{2+}-\mathrm{CaM}$ is significant in maintaining the low activity of TRPV 3 and that the calciumdependent inhibition of TRPV3 function is attenuated upon repetitive stimulation (Xiao et al., 2008a).

It has been shown that ATP can interact with TRPV1-ARD (Lishko et al., 2007). The similar nucleotide specificities of TRPV1-ARD, TRPV3-ARD, and TRPV4-ARD suggest that ATP may interact with ARD domains at a conserved binding site. Two residues in TRPV3, Lys169, and Lys174, also conserved in the ATP/CaM binding on the ARD of TRPV1, 


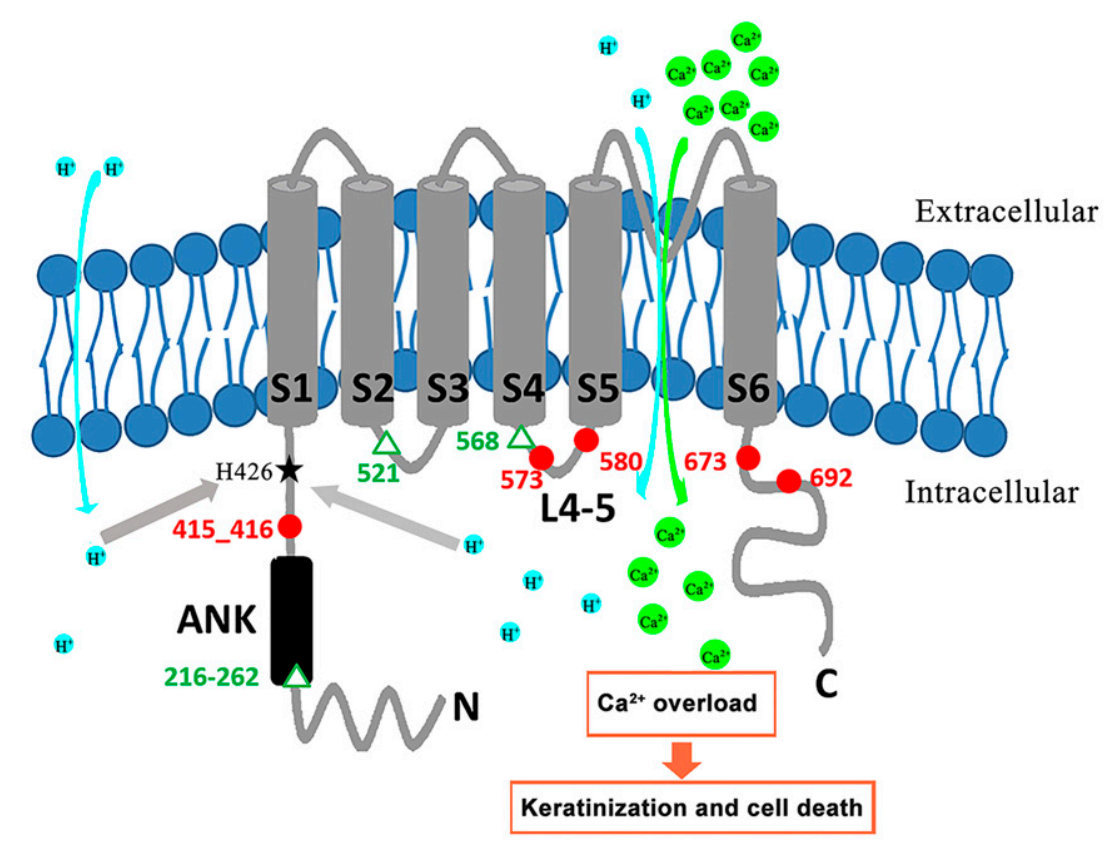

Fig. 2. A proposed model for overactive TRPV3 function or activation of TRPV3 by intracellular protons that leads to cell death and skin diseases. Schematic structure of overactive TRPV3 protein with multiple gain-of-function mutations identified from Olmsted syndrome. The solid circles in red represent dominant mutations Asn415_Arg416insLeuAsn, Gly573Cys, Gly573Ser, Gly573Ala, Gln580Pro, Leu673Phe, Trp692Phe, and Trp692Cys (Lin et al., 2012), whereas the green triangles are recessive mutations Gln216-Gly262Del, Trp521Ser, and Gly568Cys (Duchatelet et al., 2014). Weak acids can diffuse, in the protonated form, across the cell membrane and subsequently re-equilibrate to release a free proton, leading to intracellular acidification and activation of TRPV3. Protons also can pass through activated TRPV3 or other proton-permeable channels to result in intracellular acidification and activation of TRPV3. The activation of TRPV3 mediates $\mathrm{Ca}^{2+}$ influx and induces $\mathrm{Ca}^{2+}$ overload in the cytoplasm, leading to keratinization and cell death or skin diseases (Cao et al., 2012). ANK, ankyrin. have been described to be important for interactions of TRPV3 ARD with ATP and $\mathrm{Ca}^{2+}$-CaM. The binding by ATP to TRPV1 attenuates desensitization. Intriguingly, the effect is the opposite for TRPV3, where ATP reduces TRPV3 sensitivity to activators and abolishes the sensitization of TRPV3 in response to repetitive 2-APB stimulation (Phelps et al., 2010).

Under certain pathologic conditions, such as type 2 diabetes mellitus, $\mathrm{Mg}^{2+}$ deficiency results in skin disorders (Chutia and Lynrah, 2015). Luo et al. (2012) reported that TRPV3 is inhibited by intracellular or extracellular $\mathrm{Mg}^{2+}$ in primary epidermal keratinocytes, and they identified two acidic residues (E679, E682) located in the inner pore region or a residue (Asp641) from the extracellular pore loop that are critical for TRPV3-mediated signaling (Luo et al., 2012). Their findings suggest that there is a tonic inhibition of epidermal TRPV3 by both intracellular and extracellular $\mathrm{Mg}^{2+}$, such that $\mathrm{Mg}^{2+}$ deficiency may cause TRPV3 sensitization for pathogenesis of multiple skin diseases (Luo et al., 2012).

As a common modulator for ion channels in the plasma membrane, phosphatidylinositol 4,5-bisphosphate $\left(\mathrm{PIP}_{2}\right)$ regulates TRPV3 activity in primary keratinocytes of human skin or HEK293 cells expressing TRPV3. Two residues, Arg696 and Lys705, in the TRP domain of TRPV3 were shown to be responsible for the inhibition of TRPV3 channel function by $\mathrm{PIP}_{2}$ that reduces the open probability of the channel (Doerner et al., 2011). Breakdown of $\mathrm{PIP}_{2}$, in response to the activation of GPCRs that stimulate phospholipase C, causes a large shift of activation voltage from 60 to $100 \mathrm{mV}$, leading to the potentiation of TRPV3 function (Doerner et al., 2011). The $\mathrm{PIP}_{2}$-mediated modulation of TRPV3 channel suggests an interesting mechanism underlying its regulation by keratinocyte signaling cascades that drive cell proliferation and secretion of paracrine and autocrine factors (Doerner et al., 2011). Through this mechanism, activating Gq/11-mediated signaling can potentiate the function of TRPV3 (Xu et al., 2006).

\section{Role of TRPV3 in Skin Physiology}

TRPV3 is most abundantly expressed in the skin keratinocytes. Several lines of evidence suggest a critical involvement of TRPV3 in cutaneous sensations, hair development, and barrier function (Cheng et al., 2010; Aijima et al., 2015; Duchatelet and Hovnanian, 2015).

Cutaneous Pain. Similar to the situation seen with itch, epidermal keratinocytes modulate the process of pain sensation (Bang et al., 2011, 2012; Huang and Chung, 2013). TRPV3-coupled signaling mechanisms may play a crucial role in cutaneous nociception in epidermal keratinocytes. Indeed, compared with wild-type controls, keratinocytes overexpressing TRPV3 exhibit larger currents as well as augmented release of prostaglandin E2 (PGE2), an algogenic and proinflammatory intercellular messenger, that in turn activates adjoining sensory afferents (Huang et al., 2008; Saito et al., 2011). In keratinocytes, TRPV3 activation or stimulation also results in the release of ATP, another algogenic substance that might be considered as a keratinocyte-derived candidate nociceptive messenger molecule (Mandadi et al., 2009; Gifford et al., 2012). Finally, it is also shown that TRPV3-mediated release of nitric oxide from keratinocytes promotes wound healing and induces pain (Yoshida et al., 2006; Miyamoto et al., 2011).

As further evidence, the ability of endogenous TRPV3 modulators to reduce pain may indicate that TRPV3 mediates the nociceptive role of keratinocytes. Both 17R-RvD1, a proresolving lipid mediator, and isopentenyl pyrophosphate can effectively reduce inflammatory pain induced by the intradermal injection of complete Freund's adjuvant in mice, whereas farnesyl pyrophosphate causes an acute irritative response (Bang et al., 2011). In addition, silencing of TRPV3 by small hairpin RNA in epidermal keratinocytes significantly abolishes these effects (Bang et al., 2010). Certain TRPV3 antagonists are now under development in clinical phase I and II clinical trials as potential analgesic agents. Therefore, TRPV3 is apparently a novel and promising target for 
analgesic therapeutic approaches. However, there are numerous questions that should be addressed in this regard: 1) Why do TRPV3-knockout mice show strong deficits in responses to innocuous and noxious heat, as well as itch, but not in pain sensation? 2) In primates, what is the function of TRPV3 expressed in DRG neurons?

Hair Growth. Hair morphogenesis is controlled by numerous growth factors that modulate the proliferation and differentiation of keratinocytes. In mice, the phenotype of wavy hair results from loss-of-function mutations occurring naturally in the transforming growth factor- $\alpha$ (TGF- $\alpha$ ) and epidermal growth factor receptor (EGFR) genes, whereas upregulation of TGF- $\alpha$ EGFR signaling leads to the hairless phenotype (Schneider et al., 2008). Interestingly, TRPV3 knockout also gives rise to phenotypes of curly whiskers and wavy hair coat in mice (Moqrich et al., 2005). On the contrary, DS-Nh mice and WBN/Kob-Ht rats, with a gain-of-function mutation, exhibit a hairless phenotype (Xiao et al., 2008b). It has been shown that TRPV3 is critical for the appropriate development of hair in rodents, as the channel forms a signaling complex with TGF- $\alpha$ /EGFR that affects hair follicle cycling and hair morphogenesis (Cheng et al., 2010). TRPV3 activation inhibits human hair growth in an assay using cocultures of human outer root sheath keratinocytes and human organ-cultured hair follicles (Borbíró et al., 2011). In addition, small interfering RNA-mediated silencing of TRPV3 effectively abrogates the cellular effects induced by TRPV3 agonists, including functional currents, reduced proliferation, elevated concentration of intracellular calcium, and apoptosis (Borbíró et al., 2011). Collectively, those findings support the notion that TRPV3 signaling is a significant factor in regulating hair growth.

Skin Barrier Formation. Skin, the largest organ in the body, functions as a protective barrier for survival of mammals. In mice, TRPV3 expression in keratinocytes contributes to skin barrier formation and maintenance. It has been shown that the skin of newborn mice lacking TRPV3 exhibits erythroderma (red color) and is scaly and dry, matching the phenotype of defective skin barrier formation in mice (Cheng et al., 2010). The mechanism is likely that activation of EGFR enhances TRPV3 channel activity, thus stimulating the release of TGF- $\alpha$ and TGF- $\alpha$ subsequently activating EGFR and then resulting in skin barrier formation. Consistent with this, TRPV3 activation by temperatures from 36 to $40^{\circ} \mathrm{C}$ can facilitate barrier recovery after mechanical skin barrier disruption (Denda et al., 2007). However, the human relevance of these findings needs to be investigated.

\section{Role of TRPV3 in Skin Inflammation}

Atopic dermatitis (AD), one of the most common inflammatory conditions of the skin, has similar characteristics with the phenotype of mice with a gain-of-function TRPV3 mutation (Gly573Ser) that causes a spontaneously developing dermatitis as well as a hairless phenotype (although at much lower penetrance) (Yamamoto-Kasai et al., 2013). In addition, transgenic overexpression of the TRPV3 channel Gly573Ser mutant in mouse keratinocytes leads to skin inflammation, pruritus, hyperkeratosis, immune cell infiltration, upregulation of cutaneous nerve growth factors, and systemic symptoms with increased proinflammatory cytokines and plasma IgE; together, this phenotype closely resembles the clinical symptoms of human $\mathrm{AD}$ (Imura et al., 2009). A genetic study shows that the same Gly573Ser mutation also contributes to the development of hapten-induced dermatitis (Takaoka et al., 2006; Yamamoto-Kasai et al., 2013). Furthermore, TRPV3 agonists (eugenol, 2-APB) or heat activation of TRPV3 in cultured keratinocytes induces the release of PGE2 and proinflammatory interleukin- $1 \alpha$. Interestingly, a number of endogenous proinflammatory molecules (including release of bradykinin, histamine, PGE2, or ATP), receptor-coupled hydrolysis of $\mathrm{PIP}_{2}$, and activation of protein kinase $\mathrm{C} \varepsilon$ can sensitize TRPV3 to warm temperatures, resulting in an autocatalytic and TRPV3-augmented cutaneous inflammation or development of thermal hyperalgesia (Huang et al., 2008; Phelps et al., 2010).

Evidence for a proinflammatory role of TRPV3 activity in chemical nociception in the periphery of rodents is provided by the effects of $17 \mathrm{R}-\mathrm{RvD} 1$, a naturally occurring lipid mediator that inhibits TRPV3-mediated activity at nanomolar to micromolar concentrations, and also reverses the thermal hypersensitivity occurring during an inflammatory response (Bang et al., 2012). TRPV3 expression is upregulated in dermal cells isolated from rosacea, a common chronic inflammatory skin disease, further supporting the important role of the TRPV3 channel in cutaneous inflammation (Sulk et al., 2012).

\section{Identification of Gain-of-Function Mutations in Human TRPV3 That Cause Olmsted Syndrome Characterized by Severe Itching and Keratoderma}

Chronic itch is a predominant and common symptom of many cutaneous disorders (e.g., atopic eczema), various systemic conditions (e.g., liver failure and end-stage renal disease), and certain neurologic and psychiatric diseases (Liu et al., 2009). Chronic itch involves a number of targets, molecules, cells, and circuits that can trigger acute and chronic itch transmission in the peripheral and central nervous systems (Bautista et al., 2014). The existing lines of evidence suggest that keratinocytes function as the "first pruriceptive transducers" and play a crucial role in cutaneous itch sensation. It has also been suggested that TRPV3 is a possible therapeutic target in pruritus (Yamamoto-Kasai et al., 2012). DS-Nh mice (Gly573 mutated to Ser) and WBN/Kob-Ht rats (Gly573 mutated to Cys) are spontaneous hairless mutant strains in rodents that develop AD-like dermatitis under normal conditions, but not under specific pathogen-free conditions (Asakawa et al., 2006; Yoshioka et al., 2009). In rodents, spontaneous dermatitis conditions share very similar characteristics that include the following (Asakawa et al., 2006; Imura et al., 2007): 1) Staphylococcus aureus can be isolated from skin lesions, 2) levels of serum interleukin-4 and IgE are increased significantly, 3) CD4bearing $\mathrm{T}$ cells and whole mast cells are increased significantly, and 4) hyperkeratosis is observed in skin lesions with dermatitis. In DS-Nh mice expressing an overactive TRPV3 mutant, expression of thymic stromal lymphopoietin, a pruritogen, is increased in keratinocytes (Wilson et al., 2013; Yamamoto-Kasai et al., 2013), indicating that TRPV3 is a dendritic cell modulator that plays a critical role in the development of allergic dermatitis. One group has shown that 
Gly573 to Ser (Nh mutation) and Gly573 to Cys (Ht mutation) are linked to hairless phenotypes and spontaneous dermatitis in mice and rats; these are two gain-of-function mutations of the trpv3 gene at a single site that cause the channel to be constitutively open (Xiao et al., 2008b).

A TRPV3-knockout experiment has indicated that TRPV3 is involved in pruritus (Yamamoto-Kasai et al., 2012). The mouse model of dry skin initiated by application of an acetone/ether/water (AEW) mixture to the rostral back twice a day results in histamine-independent spontaneous scratching/ itch. The AEW treatment causes dry skin in both wild-type and TRPV3 knockout mice; however, spontaneous scratching is significantly increased in the AEW treated areas only in the wild-type mice, and not in TRPV3 knockout mice, demonstrating a critical role of TRPV3 in itch sensation induced by dry skin (Yamamoto-Kasai et al., 2012). However, there are some unaddressed questions: 1) If TRPV3 point mutations are responsible for the pruritus, why does no dermatitis arise on specific pathogen-free conditions? 2) Can a TRPV3-specific antagonist reduce the pruritus? 3) Why are there no reports that the TRPV3 agonists cause pruritus? 4) Is TRPV3 significantly elevated in the skin of pruritus patients? 5) Since TRPV3 is most abundantly expressed in skin keratinocytes, how is the itch information transmitted to sensory neurons?

In our collaboration with dermatologists of the Yang laboratory, three gain-of-function TRPV3 mutations from patients with Olmsted syndrome were identified. OS, also known as mutilating palmoplantar keratoderma with periorificial keratotic plaques, is a rare congenital disorder characterized by palmoplantar and periorificial keratoderma, alopecia in most cases, and severe itching (Lai-Cheong et al., 2012; Lin et al., 2012). The three gain-of-function mutations were identified from six unrelated Olmsted syndrome patients in China, in which five patients carried either the G573S (an identical mutation with the rodent) or G573C mutation, and one had a mutation at W692G (Lin et al., 2012). Whole-cell or inside-out patch clamp recordings of TRPV3 currents from HEK293 cells transfected with the TRPV3 mutants demonstrate that these mutants are constitutively open and induce large inward currents, as compared with the wild-type TRPV3 that is only an outward rectifier. The G573S or W692G mutant also elevates intracellular $\mathrm{Ca}^{2+}$ concentrations (Lin et al., 2012). The G573S mutation was also identified in a patient from India and new G573A, W692C, and L673F mutations and a homozygous recessive mutation W521S have also recently been reported for patients characterized by severe itching from Belgium, Iranian, France, and Israel (Duchatelet et al., 2014; Eytan et al., 2014; Kariminejad et al., 2014; Agarwala et al., 2016). Moreover, in one patient a heterozygous six-nucleotide insertion in TRPV3 gene resulted in the in-frame insertion of two additional amino acids (p.Asn415_Arg416insLeuAsn) (Agarwala et al., 2016). A similar skin disease named focal paloplantar keratoderma, which is characterized by the presence of circumscribed calluses on the palms and soles, also carries a causative mutation Q580P in a three-generation Chinese family (He et al., 2015).

Molecular considerations regarding the observed human mutations are as follows. The residue G573 is located in the linker region between transmembrane $\mathrm{S} 4$ and S5 segment of TRPV3 subunit, and W692 is located in the conserved TRP box (amino acid sequence of IWRLQR) of TRP domain after S6 segment in the $\mathrm{C}$ terminus. The $\mathrm{S} 4$ and $\mathrm{S} 5$ linker functions to translate movement of the voltage sensor into the gating of the pore (Liao et al., 2013). The TRP box interacts with the S4-S5 linker and couples stimulus sensing to channel gating (García-Sanz et al., 2007; Cao et al., 2013; Liao et al., 2013). Therefore, the identified three mutations located in the S4-S5 linker (G573S, G573C) or TRP domain (W692G) cause a disruption of the gating coupling and lock the channel in an open conformation. The excessive $\mathrm{Ca}^{2}$ influx through constitutively open gain-of-function mutations of TRPV3 leads to intolerant itching sensation and severe keratoderma with profound mast cell infiltration and histamine release in the upper dermis, as detected by skin biopsy sectioning (Lin et al., 2012). The nature of the gain-of-function mutations indicates that selectively targeting TRPV3 is likely an effective approach for the treatment of skin keratinization, hair loss, and itching disorders.

\section{Conclusions and Perspectives}

Since TRPV3 was first cloned in 2002, we have gained considerable insight into the structure, function, and modulation of the channel. TRPV3, the dominant TRP molecule in the skin, is involved in skin barrier formation and hair growth and mediates the cutaneous sensation of itch and pain. The identification of genetic gain-of-function mutations of TRPV3 from patients with Olmsted syndrome has significantly advanced our understanding of the causative role of dysfunctional TRPV3 in severe itching and skin diseases. The existing studies indicate that TRPV3 stands out as an attractive emerging target for chronic itch and itch-related skin diseases. Future efforts should be devoted to not only understand the molecular mechanism underlying TRPV3-mediated itch signaling but to also screen and identify specific TRPV3 antagonists that can selectively inhibit overactive TRPV3. Such agents could provide a platform for developing effective antipruritic therapies for chronic itch or TRPV3-related skin diseases.

\section{Acknowledgments}

The authors thank members of their laboratory and Dr. Yong Yang's laboratory for original contributions of TRPV3 research work. K.W. thanks J.M. Wang for consistent support.

\section{Authorship Contributions}

Wrote or contributed to the writing of the manuscript: G. Wang and $\mathrm{K}$. Wang.

\section{References}

Agarwala MK, George R, Pramanik R, and McGrath JA (2016) Olmsted syndrome in an Indian male with a new de novo mutation in TRPV3. $\mathrm{Br} J$ Dermatol 174: 209-211.

Ahmed MK, Takumida M, Ishibashi T, Hamamoto T, and Hirakawa K (2009) Expression of transient receptor potential vanilloid (TRPV) families $1,2,3$ and 4 in the mouse olfactory epithelium. Rhinology 47:242-247.

Aijima R, Wang B, Takao T, Mihara H, Kashio M, Ohsaki Y, Zhang JQ, Mizuno A, Suzuki M, Yamashita Y, et al. (2015) The thermosensitive TRPV3 channel contributes to rapid wound healing in oral epithelia. FASEB J 29:182-192.

Asakawa M, Yoshioka T, Matsutani T, Hikita I, Suzuki M, Oshima I, Tsukahara K, Arimura A, Horikawa T, Hirasawa T, et al. (2006) Association of a mutation in TRPV3 with defective hair growth in rodents. J Invest Dermatol 126:2664-2672. Auer-Grumbach M, Olschewski A, Papić L, Kremer H, McEntagart ME, Uhrig S, Fischer C, Fröhlich E, Bálint Z, Tang B, et al. (2010) Alterations in the ankyrin domain of TRPV4 cause congenital distal SMA, scapuloperoneal SMA and HMSN2C. Nat Genet 42:160-164.

Bang S, Yoo S, Yang TJ, Cho H, and Hwang SW (2010) Farnesyl pyrophosphate is a novel pain-producing molecule via specific activation of TRPV3. J Biol Chem 285: 19362-19371.

Bang S, Yoo S, Yang TJ, Cho H, and Hwang SW (2011) Isopentenyl pyrophosphate is a novel antinociceptive substance that inhibits TRPV3 and TRPA1 ion channels. Pain 152:1156-1164. 
Bang S, Yoo S, Yang TJ, Cho H, and Hwang SW (2012) 17(R)-resolvin D1 specifically inhibits transient receptor potential ion channel vanilloid 3 leading to peripheral antinociception. Br J Pharmacol 165:683-692.

Bautista DM, Wilson SR, and Hoon MA (2014) Why we scratch an itch: the molecules, cells and circuits of itch. Nat Neurosci 17:175-182.

Borbíró I, Lisztes E, Tóth BI, Czifra G, Oláh A, Szöllosi AG, Szentandrássy N, Nánási PP, Péter Z, Paus R, et al. (2011) Activation of transient receptor potential vanilloid-3 inhibits human hair growth. J Invest Dermatol 131:1605-1614.

Cao E, Liao M, Cheng Y, and Julius D (2013) TRPV1 structures in distinct conformations reveal activation mechanisms. Nature 504:113-118.

Cao X, Yang F, Zheng J, and Wang K (2012) Intracellular proton-mediated activation of TRPV3 channels accounts for the exfoliation effect of $\alpha$-hydroxyl acids on keratinocytes. J Biol Chem 287:25905-25916.

Cheng X, Jin J, Hu L, Shen D, Dong XP, Samie MA, Knoff J, Eisinger B, Liu ML, Huang SM, et al. (2010) TRP channel regulates EGFR signaling in hair morphogenesis and skin barrier formation. Cell 141:331-343.

Chung MK, Güler AD, and Caterina MJ (2005) Biphasic currents evoked by chemical or thermal activation of the heat-gated ion channel, TRPV3. J Biol Chem 280 : 15928-15941.

Chung MK, Lee H, Mizuno A, Suzuki M, and Caterina MJ (2004) 2-aminoethoxydiphenyl borate activates and sensitizes the heat-gated ion channel TRPV3. $J$ Neurosci 24:5177-5182.

Chutia H and Lynrah KG (2015) Association of serum magnesium deficiency with insulin resistance in type 2 diabetes mellitus. J Lab Physicians 7:75-78.

Deering-Rice CE, Mitchell VK, Romero EG, Abdel Aziz MH, Ryskamp DA, Križaj D, Gopal VR, and Reilly CA (2014) Drofenine: a 2-APB analogue with greater selectivity for human TRPV3. Pharmacol Res Perspect 2:e0062.

Denda M, Sokabe T, Fukumi-Tominaga T, and Tominaga M (2007) Effects of skin surface temperature on epidermal permeability barrier homeostasis. $J$ Invest Dermatol 127:654-659.

Doerner JF, Hatt H, and Ramsey IS (2011) Voltage- and temperature-dependent activation of TRPV3 channels is potentiated by receptor-mediated PI(4,5)P2 hydrolysis. J Gen Physiol 137:271-288.

Duchatelet S and Hovnanian A (2015) Olmsted syndrome: clinical, molecular and therapeutic aspects. Orphanet J Rare Dis 10:33.

Duchatelet S, Pruvost S, de Veer S, Fraitag S, Nitschké P, Bole-Feysot C, Bodemer C, and Hovnanian A (2014) A new TRPV3 missense mutation in a patient with Olmsted syndrome and erythromelalgia. JAMA Dermatol 150:303-306.

Earley S, Gonzales AL, and Garcia ZI (2010) A dietary agonist of transient receptor potential cation channel V3 elicits endothelium-dependent vasodilation. $\mathrm{Mol}$ Pharmacol 77:612-620.

Eytan O, Fuchs-Telem D, Mevorach B, Indelman M, Bergman R, Sarig O, Goldberg I, Adir N, and Sprecher E (2014) Olmsted syndrome caused by a homozygous recessive mutation in TRPV3. J Invest Dermatol 134:1752-1754.

Ferrer-Montiel A, Fernández-Carvajal A, Planells-Cases R, Fernández-Ballester G, González-Ros JM, Messeguer A, and González-Muñiz R (2012) Advances in modulating thermosensory TRP channels. Expert Opin Ther Pat 22:999-1017.

Gao L, Yang P, Qin P, Lu Y, Li X, Tian Q, Li Y, Xie C, Tian JB, Zhang C, et al. (2016) Selective potentiation of 2-APB-induced activation of TRPV1-3 channels by acid Sci Rep 6:20791.

García-Martínez C, Morenilla-Palao C, Planells-Cases R, Merino JM, and FerrerMontiel A (2000) Identification of an aspartic residue in the P-loop of the vanilloid receptor that modulates pore properties. J Biol Chem 275:32552-32558.

García-Sanz N, Valente P, Gomis A, Fernández-Carvajal A, Fernández-Ballester G, Viana F, Belmonte C, and Ferrer-Montiel A (2007) A role of the transient receptor potential domain of vanilloid receptor I in channel gating. $J$ Neurosci 27: $11641-11650$

Gifford JR, Heal C, Bridges J, Goldthorpe S, and Mack GW (2012) Changes in dermal interstitial ATP levels during local heating of human skin. $J$ Physiol 590: 6403-6411.

He Y, Zeng K, Zhang X, Chen Q, Wu J, Li H, Zhou Y, Glusman G, Roach J, Etheridge A, et al. (2015) A gain-of-function mutation in TRPV3 causes focal palmoplantar keratoderma in a Chinese family. J Invest Dermatol 135:907-909.

Holzer P and Izzo AA (2014) The pharmacology of TRP channels. Br J Pharmacol 141:2469-2473.

Hu H, Grandl J, Bandell M, Petrus M, and Patapoutian A (2009) Two amino acid residues determine 2-APB sensitivity of the ion channels TRPV3 and TRPV4. Proc Natl Acad Sci USA 106:1626-1631.

Hu HZ, Gu Q, Wang C, Colton CK, Tang J, Kinoshita-Kawada M, Lee LY, Wood JD, and Zhu MX (2004) 2-aminoethoxydiphenyl borate is a common activator of TRPV1, TRPV2, and TRPV3. J Biol Chem 279:35741-35748.

Hu HZ, Xiao R, Wang C, Gao N, Colton CK, Wood JD, and Zhu MX (2006) Potentiation of TRPV3 channel function by unsaturated fatty acids. J Cell Physiol $\mathbf{2 0 8}$ 201-212.

Huang SM and Chung MK (2013) Targeting TRPV3 for the development of novel analgesics. Open Pain J 6:119-126.

Huang SM, Lee H, Chung MK, Park U, Yu YY, Bradshaw HB, Coulombe PA, Walker JM, and Caterina MJ (2008) Overexpressed transient receptor potential vanilloid 3 ion channels in skin keratinocytes modulate pain sensitivity via prostaglandin E2. J Neurosci 28:13727-13737.

Imura K, Yoshioka T, Hikita I, Tsukahara K, Hirasawa T, Higashino K, Gahara Y, Arimura A, and Sakata T (2007) Influence of TRPV3 mutation on hair growth cycle in mice. Biochem Biophys Res Commun 363:479-483.

Imura K, Yoshioka T, Hirasawa T, and Sakata T (2009) Role of TRPV3 in immune response to development of dermatitis. J Inflamm (Lond) 6:17.

Inada H, Procko E, Sotomayor M, and Gaudet R (2012) Structural and biochemical consequences of disease-causing mutations in the ankyrin repeat domain of the human TRPV4 channel. Biochemistry 51:6195-6206.

Jin X, Touhey J, and Gaudet R (2006) Structure of the N-terminal ankyrin repeat domain of the TRPV2 ion channel. J Biol Chem 281:25006-25010.
Kariminejad A, Barzegar M, Abdollahimajd F, Pramanik R, and McGrath JA (2014) Olmsted syndrome in an Iranian boy with a new de novo mutation in TRPV3. Clin Exp Dermatol 39:492-495.

Klein AH, Carstens MI, and Carstens E (2013) Eugenol and carvacrol induce temporally desensitizing patterns of oral irritation and enhance innocuous warmth and noxious heat sensation on the tongue. Pain 154:2078-2087.

Klein AH, Trannyguen M, Joe CL, Iodi CM, and Carstens E (2015) Thermosensitive transient receptor potential (TRP) channel agonists and their role in mechanical, thermal and nociceptive sensations as assessed using animal models. Chemosens Percept 8:96-108.

Klein AS, Tannert A, and Schaefer M (2014) Cholesterol sensitises the transient receptor potential channel TRPV3 to lower temperatures and activator concentrations. Cell Calcium 55:59-68.

Lai-Cheong JE, Sethuraman G, Ramam M, Stone K, Simpson MA, and McGrath JA (2012) Recurrent heterozygous missense mutation, p.Gly573Ser, in the TRPV3 gene in an Indian boy with sporadic Olmsted syndrome. $\mathrm{Br} J$ Dermatol 167: $440-442$

Laing RJ and Dhaka A (2016) ThermoTRPs and pain. Neuroscientist 22:171-187.

Liao M, Cao E, Julius D, and Cheng Y (2013) Structure of the TRPV1 ion channel determined by electron cryo-microscopy. Nature 504:107-112.

Lin Z, Chen Q, Lee M, Cao X, Zhang J, Ma D, Chen L, Hu X, Wang H, Wang X, et al. (2012) Exome sequencing reveals mutations in TRPV3 as a cause of Olmsted syndrome. Am J Hum Genet 90:558-564.

Lishko PV, Procko E, Jin X, Phelps CB, and Gaudet R (2007) The ankyrin repeats of TRPV1 bind multiple ligands and modulate channel sensitivity. Neuron $\mathbf{5 4}$ 905-918.

Liu Q, Tang Z, Surdenikova L, Kim S, Patel KN, Kim A, Ru F, Guan Y, Weng HJ, Geng Y, et al. (2009) Sensory neuron-specific GPCR Mrgprs are itch receptors mediating chloroquine-induced pruritus. Cell 139:1353-1365.

Luo J, Stewart R, Berdeaux R, and Hu H (2012) Tonic inhibition of TRPV3 by Mg2+ in mouse epidermal keratinocytes. J Invest Dermatol 132:2158-2165.

Ma HT, Patterson RL, van Rossum DB, Birnbaumer L, Mikoshiba K, and Gill DL (2000) Requirement of the inositol trisphosphate receptor for activation of storeoperated Ca2 + channels. Science 287:1647-1651.

Ma L, Lee BH, Clifton H, Schaefer S, and Zheng J (2015) Nicotinic acid is a common regulator of heat-sensing TRPV1-4 ion channels. Sci Rep 5:8906.

Macpherson LJ, Hwang SW, Miyamoto T, Dubin AE, Patapoutian A, and Story GM (2006) More than cool: promiscuous relationships of menthol and other sensory compounds. Mol Cell Neurosci 32:335-343.

Mandadi S, Sokabe T, Shibasaki K, Katanosaka K, Mizuno A, Moqrich A, Patapoutian A, Fukumi-Tominaga T, Mizumura K, and Tominaga M (2009) TRPV3 in keratinocytes transmits temperature information to sensory neurons via ATP. Pflugers Arch 458:1093-1102.

McCleverty CJ, Koesema E, Patapoutian A, Lesley SA, and Kreusch A (2006) Crystal structure of the human TRPV2 channel ankyrin repeat domain. Protein Sci 15: $2201-2206$

Miyamoto T, Petrus MJ, Dubin AE, and Patapoutian A (2011) TRPV3 regulates nitric oxide synthase-independent nitric oxide synthesis in the skin. Nat Commun 2:369.

Moiseenkova-Bell VY and Wensel TG (2009) Hot on the trail of TRP channel structure. J Gen Physiol 133:239-244.

Moqrich A, Hwang SW, Earley TJ, Petrus MJ, Murray AN, Spencer KS, Andahazy M, Story GM, and Patapoutian A (2005) Impaired thermosensation in mice lacking TRPV3, a heat and camphor sensor in the skin. Science 307: 1468-1472.

Moussaieff A, Rimmerman N, Bregman T, Straiker A, Felder CC, Shoham S, Kashman Y, Huang SM, Lee H, Shohami $\mathrm{E}$, et al (2008) Incensole acetate, an incense component, elicits psychoactivity by activating TRPV3 channels in the brain. FASEB J 22:3024-3034.

Nilius B, Talavera K, Owsianik G, Prenen J, Droogmans G, and Voets T (2005) Gating of TRP channels: a voltage connection? J Physiol 567:35-44.

Ortar G, Morera L, Moriello AS, Morera E, Nalli M, Di Marzo V, and De Petrocellis L (2012) Modulation of thermo-transient receptor potential (thermo-TRP) channels by thymol-based compounds. Bioorg Med Chem Lett 22:3535-3539.

Peier AM, Reeve AJ, Andersson DA, Moqrich A, Earley TJ, Hergarden AC, Story GM, Colley S, Hogenesch JB, McIntyre P, et al. (2002) A heat-sensitive TRP channel expressed in keratinocytes. Science 296:2046-2049.

Phelps CB, Huang RJ, Lishko PV, Wang RR, and Gaudet R (2008) Structural analyses of the ankyrin repeat domain of TRPV6 and related TRPV ion channels. Biochemistry 47:2476-2484.

Phelps CB, Wang RR, Choo SS, and Gaudet R (2010) Differential regulation of TRPV1, TRPV3, and TRPV4 sensitivity through a conserved binding site on the ankyrin repeat domain. J Biol Chem 285:731-740.

Saito S, Fukuta N, Shingai R, and Tominaga M (2011) Evolution of vertebrate transient receptor potential vanilloid 3 channels: opposite temperature sensitivity between mammals and western clawed frogs. PLoS Genet 7:e1002041.

Schneider MR, Werner S, Paus R, and Wolf E (2008) Beyond wavy hairs: the epidermal growth factor receptor and its ligands in skin biology and pathology. Am J Pathol 173:14-24.

Schöll I and Jensen-Jarolim E (2004) Allergenic potency of spices: hot, medium hot, or very hot. Int Arch Allergy Immunol 135:247-261.

Sherkheli MA, Benecke H, Doerner JF, Kletke O, Vogt-Eisele AK, Gisselmann G, and Hatt H (2009) Monoterpenoids induce agonist-specific desensitization of transien receptor potential vanilloid-3 (TRPV3) ion channels. J Pharm Pharm Sci 12:116 128.

Sherkheli MA, Gisselmann G, and Hatt H (2012) Supercooling agent icilin blocks a warmth-sensing ion channel TRPV3. Sci World J 2012:982725.

Sherkheli MA, Vogt-Eisele AK, Weber K, and Hatt H (2013) Camphor modulates TRPV3 cation channels activity by interacting with critical pore-region cysteine residues. Pak J Pharm Sci 26:431-438. 
Smith GD, Gunthorpe MJ, Kelsell RE, Hayes PD, Reilly P, Facer P, Wright JE, Jerman JC, Walhin JP, Ooi L, et al. (2002) TRPV3 is a temperature-sensitive vanilloid receptor-like protein. Nature 418:186-190.

Stotz SC, Vriens J, Martyn D, Clardy J, and Clapham DE (2008) Citral sensing by transient [corrected] receptor potential channels in dorsal root ganglion neurons. PLoS One 3:e2082.

Sulk M, Seeliger S, Aubert J, Schwab VD, Cevikbas F, Rivier M, Nowak P, Voegel JJ, Buddenkotte J, and Steinhoff M (2012) Distribution and expression of nonneuronal transient receptor potential (TRPV) ion channels in rosacea. J Invest Dermatol 132:1253-1262.

Takaoka A, Arai I, Sugimoto M, Honma Y, Futaki N, Nakamura A, and Nakaike S (2006) Involvement of IL-31 on scratching behavior in NC/Nga mice with atopiclike dermatitis. Exp Dermatol 15:161-167.

Wilson SR, Thé L, Batia LM, Beattie K, Katibah GE, McClain SP, Pellegrino M, Estandian DM, and Bautista DM (2013) The epithelial cell-derived atopic dermatitis cytokine TSLP activates neurons to induce itch. Cell 155:285-295.

Xiao R, Tang J, Wang C, Colton CK, Tian J, and Zhu MX (2008a) Calcium plays a central role in the sensitization of TRPV3 channel to repetitive stimulations. J Biol Chem 283:6162-6174.

Xiao R, Tian J, Tang J, and Zhu MX (2008b) The TRPV3 mutation associated with the hairless phenotype in rodents is constitutively active. Cell Calcium 43:334-343.

$\mathrm{Xu}$ H, Blair NT, and Clapham DE (2005) Camphor activates and strongly desensitizes the transient receptor potential vanilloid subtype 1 channel in a vanilloidindependent mechanism. $J$ Neurosci 25:8924-8937.

Xu H, Delling M, Jun JC, and Clapham DE (2006) Oregano, thyme and clove-derived flavors and skin sensitizers activate specific TRP channels. Nat Neurosci 9:628-635.
Xu H, Ramsey IS, Kotecha SA, Moran MM, Chong JA, Lawson D, Ge P, Lilly J, SilosSantiago I, Xie Y, et al. (2002) TRPV3 is a calcium-permeable temperaturesensitive cation channel. Nature 418:181-186.

Yamamoto-Kasai E, Imura K, Yasui K, Shichijou M, Oshima I, Hirasawa T, Sakata T, and Yoshioka T (2012) TRPV3 as a therapeutic target for itch. J Invest Dermatol 132:2109-2112.

Yamamoto-Kasai E, Yasui K, Shichijo M, Sakata T, and Yoshioka T (2013) Impact of TRPV3 on the development of allergic dermatitis as a dendritic cell modulator. Exp Dermatol 22:820-824.

Yoshida T, Inoue R, Morii T, Takahashi N, Yamamoto S, Hara Y, Tominaga M, Shimizu S, Sato Y, and Mori Y (2006) Nitric oxide activates TRP channels by cysteine S-nitrosylation. Nat Chem Biol 2:596-607.

Yoshioka T, Imura K, Asakawa M, Suzuki M, Oshima I, Hirasawa T, Sakata T, Horikawa T, and Arimura A (2009) Impact of the Gly573Ser substitution in TRPV3 on the development of allergic and pruritic dermatitis in mice. $J$ Invest Dermatol 129:714-722.

Zhang X (2015) Targeting TRP ion channels for itch relief. Naunyn Schmiedebergs Arch Pharmacol 388:389-399.

Address correspondence to: KeWei Wang, Department of Pharmacology, Qingdao University School of Pharmacy and Institute of Innovative Drugs, Qingdao University, 38 Dengzhou Road, Qingdao 266021, Shandong Province, China. E-mail: wangkw@qdu.edu.cn 